\title{
Application of Fuzzy-Flower Pollination Algorithmfor Peak Load Forecasting on National Holiday
}

\author{
Andi Imran ${ }^{\# 1}$, I Made Yulistya Negara ${ }^{\# 2}$, Imam Robandi ${ }^{\# 3}$ \\ \#Department of Electrical Engineering, Sepuluh Nopember Institute of Technology, \\ Sukolilo Campus, Surabaya, Indonesia \\ ${ }^{1}$ andi.imran13@mhs.ee.its.ac.id \\ ${ }^{2}$ yulistya@ee.its.ac.id \\ 3 robandi@ee.its.ac.id
}

\begin{abstract}
Application of Type-2 Fuzzy Logic System (T2FLS) has became attention for a short-term load forecasting problems solution. This paper presentsapplication optimization membership function of antecedent $(X, Y)$ and consequent $(Z)$ interval type-2 Fuzzy Logic System using Flower Pollination Algorithm (FPA) for short-term load forecasting on national holiday. This method has being implemented on the historical peak load data during 14 national holidays case study in JawaBaliIndonesia electrical power system in 2011. Flower Pollination Algorithm (FPA) will be applied to optimize interval Footprint of Uncertainty (FOU) membership functions of interval type-2 fuzzy logic system. The test result showed Main Absolute Percentage Error (MAPE) is less than type-2 Fuzzy Logic System (FLS) and optimization type-2 FLS-Big Bang Big Crunch Algorithm. Finally, this paper defined Main Absolute Percentage Error (MAPE) $\mathbf{2 . 0 4 0 6 1 2 1 4 3 \%}$ for type-2 FLS, $\mathbf{1 . 2 7 9 2 5 7 1 4 3 \%}$ for optimization type-2 FLS-Big Bang Big Crunch Atgorithm and 1.091543571\% for optimization type2 FLS-Flower Pollination Algorithm.
\end{abstract}

Keywords:Type-2 Fuzzy Logic, Flower Pollination Algorithm, MAPE, Membership Function, National Holiday

\section{INTRODUCTION}

The most important thing in electric power transmissionsystem isthe precise calculation of power generation that meet a certain required load [1]. Requiredload at certainfine can be solved by load forecast technique. Load forecastingis classifiedintothree categoriesthat areshort-term load forecasting,mid-term load forecasting andlong-term load forecasting [2]. Research on load forecastingbecomesvery importantin modern countryespeciallyshort-term load forecasting becausethe appearance ofenergy marketwhich is very competitive [2]. Load forecasting on holiday becomes an interest because itdiffers from ordinary days [3]. Arenormally implemented in load forecasting that is conventional method and intelligent method $[4,5]$. Conventional method isstatistical method.A complex nonlinear system with series uncertainty factor is difficult to solve using conventional method. Sometimes it leads to high inaccurate of load forecasting [5]. Intelligent method hasabilityto givebetter performance in handling non-linear problem[5]. Intelligent methodwhich is often usedon load forecastingthat is Artificial Neural Network (ANN) [6-9] and fuzzy logic [10-14]. The advantageof ANN isits abilitytolearnthe historical load pattern. However, conventional ANN model sometimes has overfitting problems which result in improper forecasting results.[15]. Moreover, it is often difficult to obtain the best ANN due to tiresome tuning and trial-and-error process [5].

On the other hand,FuzzyLogic (FL) providessimple way tosolvesome drawbacks which are feedback which is vague, ambiguous, inexact, noise, or missing information to get on exact conclusion. Linguisticvariableis usedtorepresent parameter of an FL system operation. FL uses "if $X$ and $Y$ then $Z$ " isan approachnotmathematical solving. It is very usefulincontrollingcomplexnonlinearsystem, which is unsolvable by mathematical model [16]. Fuzzy logic is foundbyProf.Zadehwhich has developed into fuzzy logic type 2 [17]. FLSs type-1 cannotdirectlyhandlethe uncertainty ofrule, because it uses certain type-1 fuzzy set (that isfullyexplainedbysingle numericvalues). On the other hand, type-2 FLSs isusefulin difficult situationto determineexact numericmembership function, andthe uncertainty ofmeasurement [18]. Type-2 FLSs can be usedin uncertainty situationto determinethe exact membership valuesuch asin training where the data is influencedby noisy [18].

In this research, authors apply fuzzy logic type 2 method which is optimized by using flower pollination algorithm in order to forecast peak load on Indonesian national holiday. Flower pollination algorithm isoptimizationmethod which is inspiredbyflower pollination process [19]. This methodis claimed more efficientthan GA and PSO [19]. 


\section{INTERVAL TYPE-2 FUZZY LOGIC}

Fuzzy logic system type 2 isthe expansionof fuzzy logic system type 1 wheremembership function of fuzzy logic system type 2 hastwo membership degrees that areprimaryandsecondary membership degrees.Fuzzylogic system type 2 consists offuzzification, a set of rules (rules), fuzzyinference machine andoutput processor. Output processoron fuzzylogic system type 2consists oftype-reduceranddefuzzification. Type-reducerchangefuzzytype-2 set intosomefuzzytype-1set; one of them usesKernik Mendel Algoritm (KMA)anddefuzzificationwhich will resultingoutput crisp (output crisp). Fuzzy logic system type 2 is alsocharacterizedbyIF-THENrule, butits antecedentandconsequencemembership setsistype-2. Generally, fuzzy logic system type 2can be seen onfigure 1.

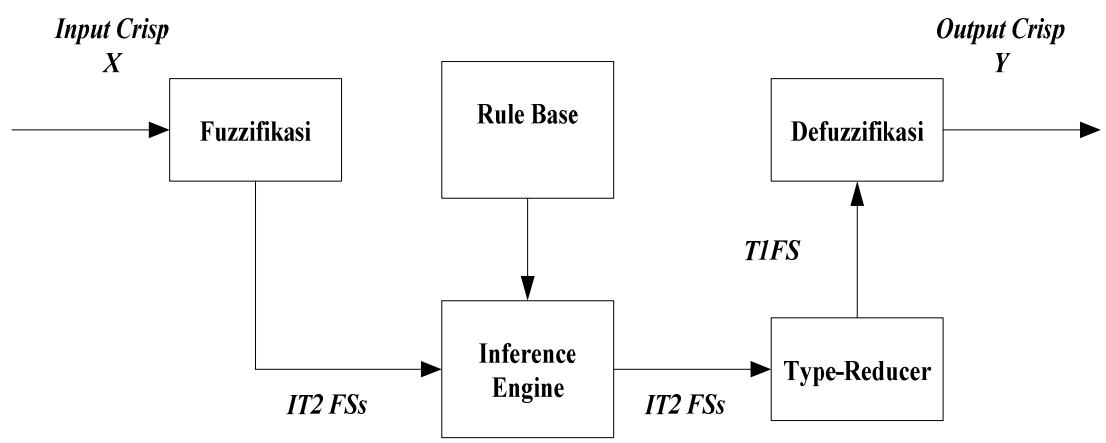

Fig 1.Type-2 Fuzzy Logic System (T2FLS) Structure [17]

\section{A. Interval Type-2 Fuzzy Set}

IntervalType-2 FuzzySet (IT2FS) is denoted $\tilde{A} . \mu \tilde{A}$ is membership function with $x \in X$ and $u \in J x \subseteq[0,1]$. Characteristicof IT2FS can be recognized on the following equation:

$$
\tilde{A}=\int_{x \in X} \int_{x \in J_{x}} \frac{\mu \widetilde{A}(x, u)}{(x, u)} J x \subseteq[0,1]
$$

Primaryvariable $\mathrm{x}$ which has domain $\mathrm{X} ; u \in U$, secondary variable, have domain $J x$ for each $x \in X$; $J x$ is

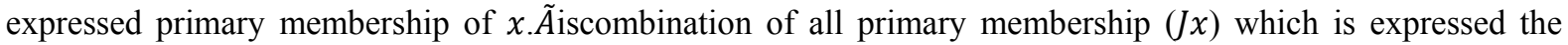
Footprint of Uncertainty (FOU) of $\tilde{A}$. The equation can be seen as follows:

$$
\operatorname{FOU}(\tilde{A})=\bigcup_{\forall x \in X} J x=\{(x, u) ; u \in J x \subseteq[0,1]\}
$$

$\mathrm{JX}$ is interval with the following equation:

$$
J x=\left\{(x, u) ; u \in\left[\underline{\mu}_{\tilde{A}}(x), \bar{\mu}_{\tilde{A}}(x)\right]\right\}
$$

From equation FOU $(\tilde{A})$ can be expressed by the equation:

$$
\operatorname{FOU}(\tilde{A})=\bigcup_{\forall x \in X}\left[\underline{\mu}_{\tilde{A}}(x), \bar{\mu}_{\tilde{A}}(x)\right]
$$

$J x=$ Primary membershipof $x$

$\mu \tilde{A}=$ Lower Membership Function (LMF) of $\tilde{A}$

$\overline{\bar{\mu}} \tilde{A}=$ Upper Membership Function (UMF) of $\tilde{A}$

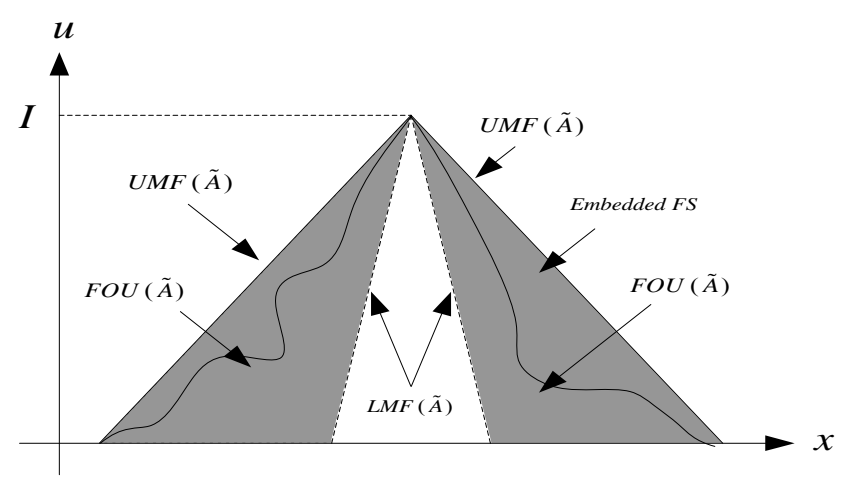

Fig 2. FOU (darkcolor), LMF (dotted line), UMF (solid line) and Embedded FS (wavy line) [17] 


\section{B. Interval Type-2 Fuzzy Membership Function Operations}

Interval type-2fuzzy set operation which is represented by FOUis doneby using two intervalsthat isUpper Membership Function (UMF)andLower Membership Function (LMF). Operation onmembership function fuzzy interval type-2can be seen on figure3:

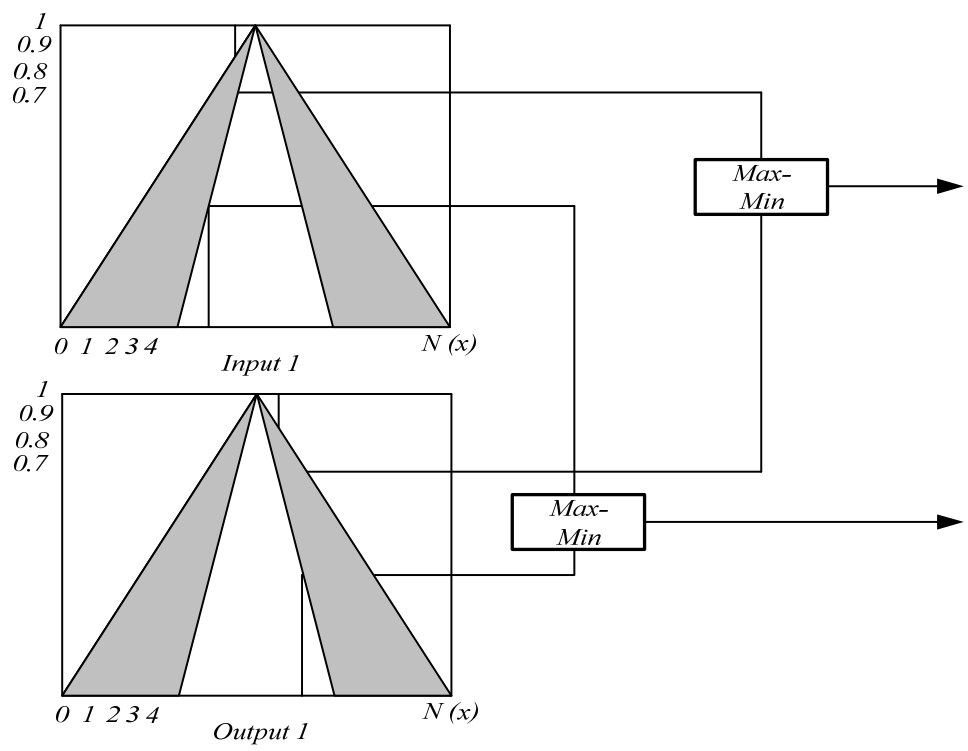

Fig 3. Operation fuzzy set interval type-2 (IT2FLS)

\section{C.Kernik Mendel Algorithm}

On interval type-2 fuzzy, processof searching the centroid can be doneby using Kernik Mendel Method.This searching methodis formulatedas follows:

$$
\begin{gathered}
Y_{\operatorname{Cos}}\left(x^{\prime}\right)=\bigcup_{\begin{array}{l}
f^{n} \in F^{n}\left(x^{\prime}\right) \\
y^{n} \in Y^{n}
\end{array}} \frac{\sum_{n-1}^{N} f^{n} y^{n}}{\sum_{n-1}^{N} f^{n}}=[y l, y r] \\
y_{1}=\min _{k \in[1, N-1]} \frac{\sum_{n=1}^{k} \bar{f}^{n} \underline{y}^{n}+\sum_{n=k+1}^{N} \underline{f}^{n} \underline{\underline{y}}^{n}}{\sum_{n=1}^{k} \overline{\bar{f}}^{n}+\sum_{n=k+1}^{N} \underline{f}^{n}} \equiv \frac{\sum_{n=1}^{L} \bar{f}^{n} \underline{y}^{n}+\sum_{n=L+1}^{N} \underline{f}^{n} \underline{\underline{y}}^{n}}{\sum_{n=1}^{L} \bar{f}^{n}+\sum_{n=L+1}^{N} \underline{f}^{n}} \\
y_{r}=\max _{k \in[1, N-1]} \frac{\sum_{n=1}^{k} \underline{f}^{n} \bar{y}^{n}+\sum_{n=k+1}^{N} \bar{f}^{n} \bar{y}^{n}}{\sum_{n=1}^{k} \underline{f}^{n}+\sum_{n=k+1}^{N} \bar{f}^{n}} \equiv \frac{\sum_{n=1}^{R} \underline{f}^{n} \bar{y}^{n}+\sum_{n=R+1}^{N} \bar{f}^{n} \bar{y}^{n}}{\sum_{n=1}^{R} \underline{f}^{n}+\sum_{n=R+1}^{N} \bar{f}^{n}}
\end{gathered}
$$

Switch point of $L$ and $R$ are as follows:

$$
\begin{aligned}
& \underline{y}^{L} \leq y l \leq \underline{y}^{L+1} \\
& \quad \bar{y}^{R} \leq y r \leq \bar{y}^{R+1}
\end{aligned}
$$

The searching ofcentroid value is doneby following equation:

$$
\text { Centroid }=\frac{(y l+y r)}{2}
$$

\section{Flower PollinationAlgorithm}

Flower Pollination Algorithm(FPA) isanoptimization methodwhich is taken based oncharacteristic of flower pollination. In using this method,there are rulesof flower pollination phenomenon characteristic, flower constancyphenomenon, andpollination behavioras follows:

1. Biotic pollinationand cross-pollinationare consideredas global pollination process where pollinatorcarriespollen (pollen-carrying) doingLévyFlightsmovement.

2. Abiotic pollination and single pollinationare considered as local pollination.

3. Flower constancy is considered aschance (probability) reproductionwhich is proportionalwith similarity from two involved flowers.

4. Local and global pollinationsare regulatedby switch probability $\in=[0,1]$

There are two fundamental things on this algorithm that is global andlocal pollinations. On global pollination, pollen from flower is carriedbyanimal pollinatorssuch asinsectandpollen can dolong-distance travelbecause insect can flyand move in large area. This processplus flower constancy phenomenon can be represented mathematically as:

$$
x_{i}^{t+1}=x_{i}^{t}+\gamma L(\lambda)\left(x_{i}^{t}-g^{*}\right)
$$


with $\mathrm{x}_{\mathrm{i}}^{\mathrm{t}}$ declarespolleniorvectorsolution $x_{i}$ oniterationt, and $g_{*}$ isthe best solutiononongoing iteration. Parameter $L$ ispollination power. Because insect can moveinto long-distancewith different step, wecan useLévyFlightstoimitatethis characteristic efficiently, that iswe take $L>0$ fromLévy distribution.

$$
L \sim \frac{\lambda \Gamma(\lambda) \sin \left(\frac{\pi \lambda}{2}\right)}{\pi} \frac{1}{s^{1+\lambda}},\left(s \gg s_{0}>0\right)
$$

$\Gamma(\lambda)$ is gamma standard function, andthis distributionis appliedtos $>0$ step. Then,local pollinationand flower constancy can be represented as:

$$
\mathrm{x}_{\mathrm{i}}^{\mathrm{t}+1}=\mathrm{x}_{\mathrm{i}}^{\mathrm{t}}+\in\left(\mathrm{x}_{\mathrm{j}}^{\mathrm{t}}-\mathrm{x}_{\mathrm{k}}^{\mathrm{t}}\right)
$$

with $x_{j}^{t}$ and $x_{k}^{t}$ arepollen from different flowersof similar plant species. This rule imitates flower constancy phenomenon in limited environment. Mathematically, if $x_{k}^{t}$ and $x_{k}^{t}$ come fromsimilar population, then this rule becomesrandom walklocalif we take $\epsilon$ from uniform distribution $[0,1]$.

\section{Peak Load Forecasting On National Holiday Using IT2FL-Flower Pollination Algorithm}

There are three steps which is done toapply fuzzy type 2-flower onpeak loadforecasting onholidaynationalthat ispre-processing, processing and post-processing [7].

\section{A. Pre-Processing}

Pre-Processingispreparation ofpeakloaddata onnationalholidaytoobtain Load Difference (LD), Typical Load Difference (TLD), Maximum Weekdays (max WD) andVariationLoadDifference(VLD). Load Difference (LD) isthe difference ofload 4 daysbeforenational holiday which is given by:

$$
\begin{aligned}
& \operatorname{LD}_{M A X}(i)=\frac{\operatorname{MaxSD}(i)-\operatorname{Max} W D(i)}{\operatorname{Max} W D(i)} \times 100 \\
& \operatorname{Max} W D_{(i)}=\frac{W D_{(i) d-4}+W D_{(i) d-3}+W D_{(i) d-2}+W D_{(i) d-1}}{4}
\end{aligned}
$$

$\operatorname{maxSD}(\mathrm{i})$ ispeak load on holidayandmaxWDisthe average of maximum load 4 daysbeforeholiday. After thatcalculate the Typical Load Difference $\left(\operatorname{TLD}_{\operatorname{MAX}}(\mathrm{i})\right)$ that isaveragingthe peak load of $\operatorname{LD}_{\operatorname{MAX}}(\mathrm{i})$ which is similarin previous year.Then looking forVariation Load Differencethat isthe differencebetween Load Difference (LD) fromTypical Load Difference $\left(\operatorname{TLD}_{\mathrm{MAX}}(\mathrm{i})\right)$ withfollowingequation:

$$
\begin{aligned}
& V L D_{\text {max }}(i)=L D_{M A X}(i)-T L D_{M A X}(i) \\
& T L D_{\text {max }}(i)=\frac{L D_{M A X}(i-1)+L D_{M A X}(i-2)+L D_{M A X}(i-3)}{3}
\end{aligned}
$$

\begin{tabular}{|c|c|c|c|c|}
\hline \multicolumn{5}{|c|}{ National Holidays Peak Load in 2013 (MW) } \\
\hline WD(i) $)_{d-4}$ & WD(i) $)_{d-3}$ & WD(i) $)_{\mathrm{d}-2}$ & WD(i) $)_{\mathrm{d}-1}$ & $\operatorname{MaxSD(i)}$ \\
\hline 19782.00 & 18608.00 & 17525.00 & 16872.00 & 15780.00 \\
\hline 17094.00 & 18296.00 & 18968.00 & 19424.00 & 17354.00 \\
\hline 22146.00 & 20961.00 & 19903.00 & 19764.00 & 18650.00 \\
\hline 21276.00 & 20643.00 & 19568.00 & 21315.00 & 19477.00 \\
\hline 18309.00 & 20350.00 & 20134.00 & 19735.00 & 18307.00 \\
\hline 19099.00 & 21123.00 & 21734.00 & 21506.00 & 19071.00 \\
\hline 17337.00 & 17151.00 & 16201.00 & 14942.00 & 13777.00 \\
\hline 17151.00 & 16201.00 & 14942.00 & 13777.00 & 14058.00 \\
\hline 21252.00 & 21380.00 & 20828.00 & 18496.00 & 18853.00 \\
\hline 18897.00 & 21910.00 & 21968.00 & 21592.00 & 19914.00 \\
\hline 20120.00 & 18429.00 & 20732.00 & 20627.00 & 18782.00 \\
\hline 20768.00 & 19744.00 & 18612.00 & 20299.00 & 18723.00 \\
\hline 20333.00 & 20730.00 & 20953.00 & 19293.00 & 17875.00 \\
\hline 21862.00 & 21677.00 & 21327.00 & 21428.00 & 18662.00 \\
\hline
\end{tabular}

To calculate Max WD and LD max based on (12) and (13) equations can be seen on Table land Table 2.

TABLE I. Peak Load In 2013

TABLE II. VLD max forIdulFitri I 2012and 2013

\begin{tabular}{|c|c|c|c|c|}
\hline Year & Max WD & LD Max & TLD max & VLD max \\
\hline 2013 & 16407.8 & -16.034 & -14.926 & -1.1077 \\
\hline 2012 & 15994.5 & -17.628 & -14.385 & -3.2425 \\
\hline
\end{tabular}




\section{B. Processing}

The operation of FLS Type-2 is similar withoperation on fuzzy type-1, but FLS Type-2 has FOU whichismembership functionthat is limitedbyUpper Membership Function (UMF) andLower Membership Function (LMF).

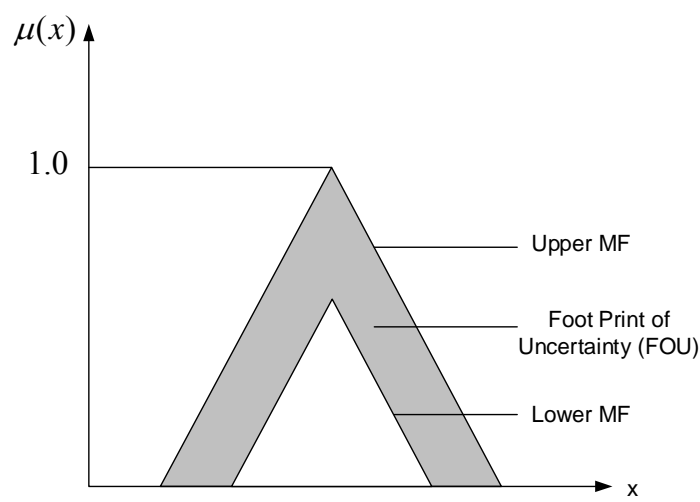

Fig4. FOU fuzzy type 2

The rule of fuzzy IF-THEN is used inthis methodtoforecastpeakloadwhich is declared as follows:

IF $X$ is $A_{i}$ AND $Y$ is $B_{i}$ THEN $Z$ is $C_{i}$

$\mathrm{X}$ and $\mathrm{Y}$ inputs byusing IT2MF Editor infuzzificationdesign, there are 11 membership functionswhich are used [7], that is:

Negative Very Big (UNVB and LNVB)

Negative Big (UNB and LNB)

Negative Medium (UNM and LNM)

Negative Small (UNS and LNS)

Negative Very Small (UNVS and LNVS)

Zero (UZE and LZE)

Positive Very Small (UPVS and LPVS)

Positive Small (UPS and LPS)

Positive Medium (UPM and LPM)

Positive Big (UPB and LPB)

Positive Very Big (UPVB and LPVB)

Examples of fuzzy rules can be seen in Table 3 .

TABLE III. Fuzzy Rules

\begin{tabular}{|c|c|c|c|}
\hline No. & \multicolumn{2}{|c|}{ Antecendent } & Consequent \\
\hline Rules & X & Y & Z \\
\hline 1 & NVS & NVS & NVS \\
\hline 2 & PVS & PVS & PVS \\
\hline 3 & ZE & NVS & NVS \\
\hline 4 & PVS & ZE & ZE \\
\hline 5 & PVS & PVS & PVS \\
\hline 6 & NVS & PM & PM \\
\hline 7 & NVS & PS & PS \\
\hline 8 & ZE & PS & PS \\
\hline 9 & ZE & ZE & ZE \\
\hline 10 & ZE & PM & PM \\
\hline 11 & NVS & NVS & NVS \\
\hline 12 & PVS & NVS & NVS \\
\hline 13 & ZE & PVS & PVS \\
\hline 14 & ZE & PVS & PVS \\
\hline
\end{tabular}


The rule of rule editor intable 3 can be seen as follows:

[R1] IF $\mathrm{X}$ is NVS AND Y is NVS THEN Z is NVS

[R2] IF $X$ is PVS AND Y is PVS THEN $Z$ is PVS

[R14] IF $\mathrm{X}$ is ZE AND Y is PVS THEN Z is PVS

In choosing fuzzy set using max rule is by taking the biggest valuewhich is appropriate withmembership degree $(\mu)$ of input variable $(X, Y)$ and output $(Z)$ on New Year can be seen inTable 4.Valuewhich is madeinto input to $\mathrm{X}, \mathrm{Y}$ and $\mathrm{Z}$ variables areVLDmaxfromholiday data.X isVLDmax (i) fromsimilar holidaybefore forecasting year. $Y$ isVLDmax (i) fromholidaywhich is adjacentin forecasting year. $Z$ is forecast of VLDmax (i). Variablevalue of $\mathrm{X}, \mathrm{Y}$ and $\mathrm{Z}$ is madeasdividerto $\mathrm{LMF}$ and $\mathrm{UMF}$ parameters. After that, parameter value of LMF and UMF on FOU is optimizedbyusing flower pollination algorithm. $\mathrm{X}, \mathrm{Y}$ and $\mathrm{Z}$ variables can be seeninfigure6,7 and 8.Flowchart of fuzzy type 2-flower pollination algorithm on peak load forecastingon national holidaycan be seen infigure 5 .

C. Post-Processing

The next process islooking forforecast load differencevalue which can be declared as follows:

$$
\text { Forecast } L D_{M A X}(i)=\text { Forecast } V L D_{M A X}(i)+T L D_{M A X}(i)
$$

Thenpeak load forecastingon national holidaycan be calculated as follows:

$$
P_{M A X}^{\prime}(i)=\operatorname{MaxWD}(i)+\frac{\left(\text { Forecast } L D_{M A X} x \operatorname{Max} W D(i)\right)}{100}
$$

To find outthe accuracyofproposed methodthenused absolute error equation. The smaller error which isobtainedindicatesthe used methodis better. Absolute error equation as follows:

$$
\begin{aligned}
& \text { Error }=\left|\frac{P_{\text {forecast }}-P_{\text {actual }}}{P_{\text {actual }}}\right| \times 100 \% \\
& \text { Error }=\left|\frac{P_{M A X}^{\prime}(i)-\operatorname{MaxSD}(i)}{\operatorname{MaxSD}(i)}\right| \times 100 \%
\end{aligned}
$$




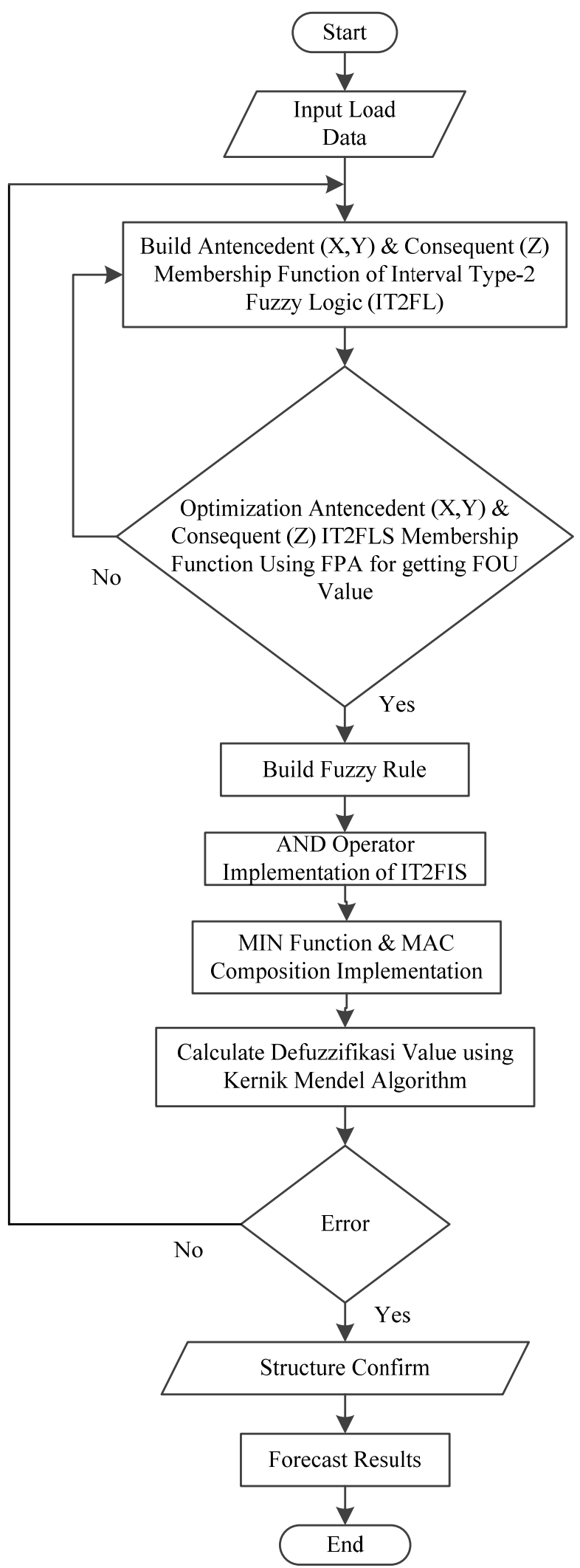

Fig5. Flowchart IT2FL-Flower for Peak Load Forecasting on National Holidays 
TABLE IV. Establishment Of Rule Base For Input X in2013

\begin{tabular}{|c|c|c|c|c|c|c|c|c|c|c|c|c|c|c|}
\hline \multirow{2}{*}{$\begin{array}{l}\text { Holidays } \\
\text { Name }\end{array}$} & \multirow{2}{*}{ Variable } & \multirow{2}{*}{ VLD max } & \multicolumn{11}{|c|}{ Membership Function $(\mu)$} & \multirow{2}{*}{$\frac{\text { Set of }}{\mathrm{X}}$} \\
\hline & & & NVB & NB & NM & NS & NVS & $\mathbf{Z E}$ & PVS & PS & PM & PB & PVB & \\
\hline \multirow{3}{*}{$\begin{array}{c}\text { Tahun } \\
\text { Baru } \\
\text { Masehi }\end{array}$} & $X$ & -3.278377375 & & & & & 0.819594344 & 0.1804 & & & & & & NVS \\
\hline & $\mathrm{Y}$ & -2.900956448 & & & & & 0.725239112 & 0.27476 & & & & & & NVS \\
\hline & $\mathrm{Z}$ & -2.900956448 & & & & & 0.725239112 & 0.27476 & & & & & & NVS \\
\hline
\end{tabular}

Antecedent (X, Y) and consequent (Z) T2FIS figures as follows:

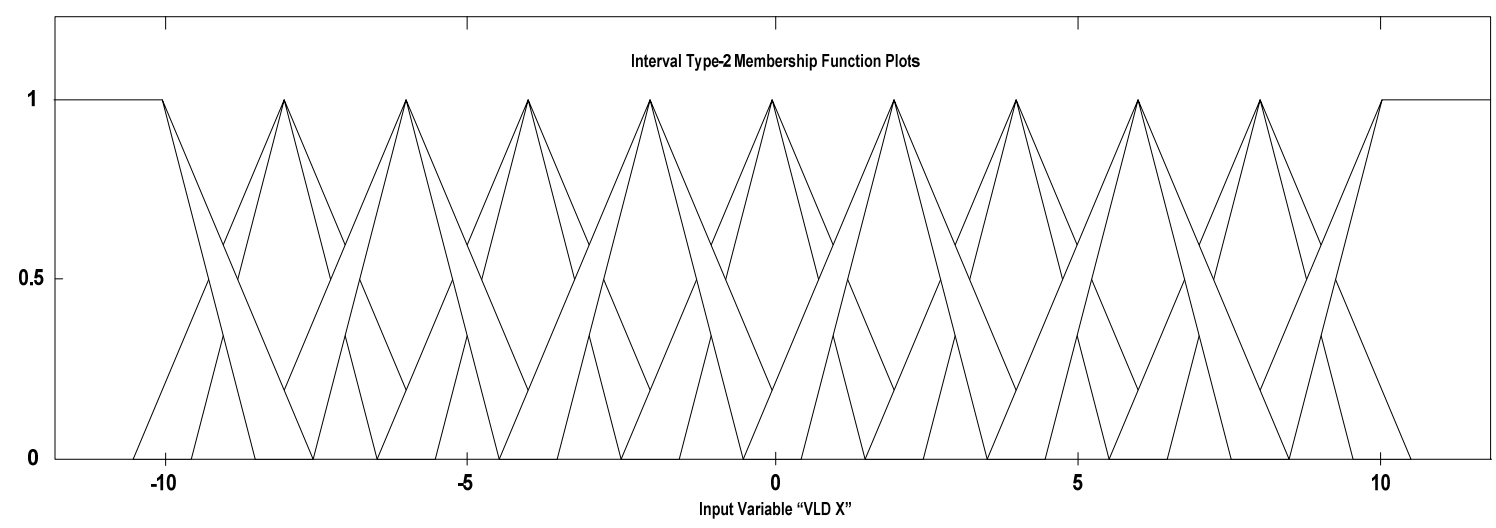

Fig6. Membership Function for Variable Input X T2FIS

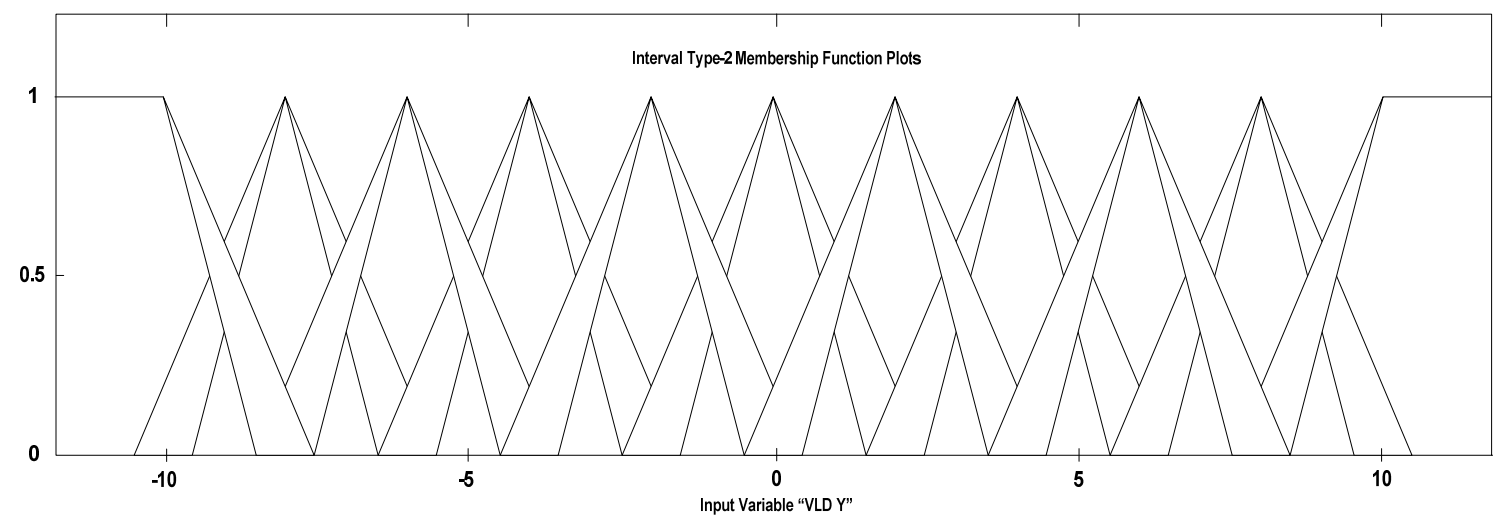

Fig7. Membership Function for Variable Input Y T2FIS

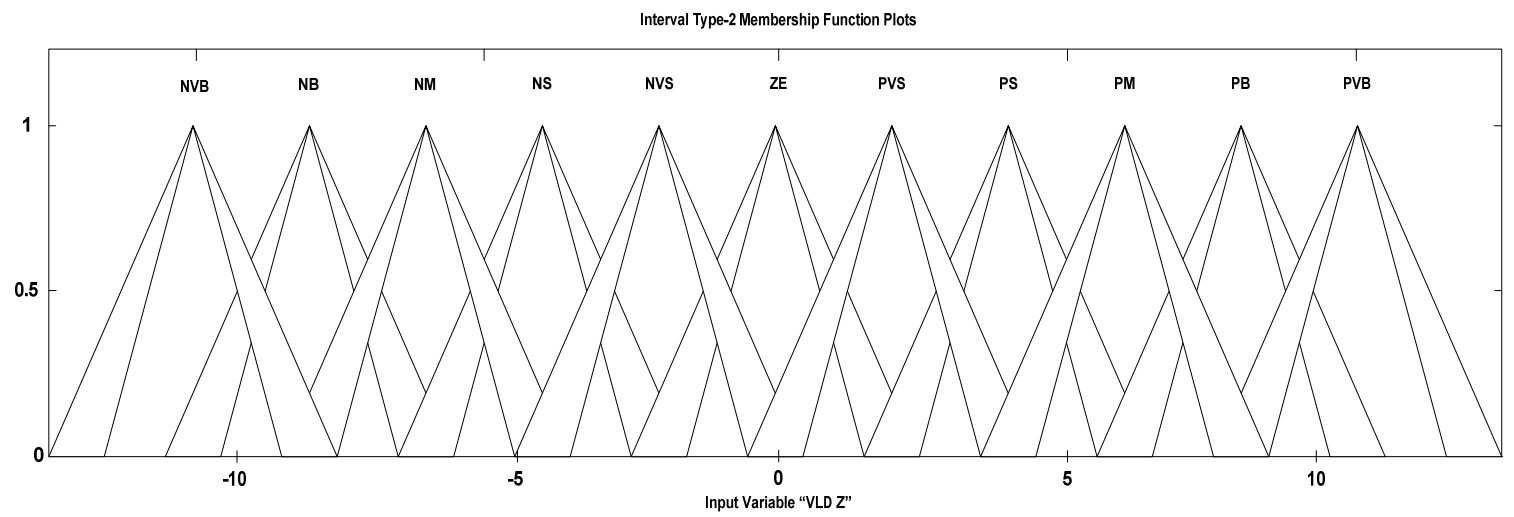

Fig8. Membership Function for Variable Input Z T2FIS 
TABLE V. Results of VLD Forecast on National Holidaysin 2013

\begin{tabular}{|c|c|c|c|c|c|c|c|c|}
\hline \multirow{2}{*}{ No } & \multirow{2}{*}{ Holidays Name } & \multirow{2}{*}{$\begin{array}{c}\text { VLD } \\
\text { Target }\end{array}$} & \multicolumn{2}{|c|}{ IT2 } & \multicolumn{2}{|c|}{ IT2-BBBC } & \multicolumn{2}{|c|}{ IT2-FPA } \\
\hline & & & $V L D$ & Error(\%) & $V L D$ & Error(\%) & $V L D$ & Error(\%) \\
\hline 1 & Tahun Baru Masehi & -0.4991174 & $-8.33 \mathrm{E}-17$ & 0.4991174 & $\begin{array}{l}2.60 \mathrm{E}- \\
06\end{array}$ & 0.49912 & $\begin{array}{c}2.40 \mathrm{E}- \\
01\end{array}$ & 0.73912 \\
\hline 2 & $\begin{array}{l}\text { Proklamasi } \\
\text { Kemerdekaan RI }\end{array}$ & $\begin{array}{c}7.7473500 \\
15 \\
\end{array}$ & $\begin{array}{c}6.3359498 \\
07 \\
\end{array}$ & $\begin{array}{c}1.4114002 \\
08\end{array}$ & 4.44 & 3.30735 & $\begin{array}{c}6.21751 \\
38 \\
\end{array}$ & 1.529836 \\
\hline 3 & Idul Adha & $\begin{array}{c}1.7980963 \\
45\end{array}$ & $\begin{array}{c}3.8370748 \\
35\end{array}$ & $\begin{array}{c}2.0389784 \\
9 \\
\end{array}$ & $\begin{array}{c}1.66141 \\
51\end{array}$ & 0.136681 & $\begin{array}{c}3.98961 \\
89\end{array}$ & 2.19152 \\
\hline 4 & Tahun Baru Hijriyah & $\begin{array}{c}1.4891024 \\
43\end{array}$ & $\begin{array}{l}2.0017456 \\
55\end{array}$ & $\begin{array}{c}0.5126432 \\
1\end{array}$ & $\begin{array}{c}1.53604 \\
4\end{array}$ & 0.04694 & $\begin{array}{c}1.42431 \\
54\end{array}$ & 0.064787 \\
\hline 5 & $\begin{array}{l}\text { Maulid Nabi } \\
\text { Muhammad SAW }\end{array}$ & $\begin{array}{c}2.1110236 \\
55 \\
\end{array}$ & $\begin{array}{c}0.4947342 \\
43\end{array}$ & $\begin{array}{c}1.6162894 \\
12 \\
\end{array}$ & $\begin{array}{c}0.40011 \\
56\end{array}$ & 1.710908 & $\begin{array}{c}0.63739 \\
16 \\
\end{array}$ & 1.473632 \\
\hline 6 & Isra Mi'raj & $\begin{array}{c}- \\
3.1637548 \\
6 \\
\end{array}$ & $\begin{array}{c}- \\
4.3819774 \\
8 \\
\end{array}$ & $\begin{array}{c}1.2182226 \\
25 \\
\end{array}$ & $\begin{array}{c}- \\
3.50451\end{array}$ & 0.340755 & $\begin{array}{c}- \\
3.10850 \\
1\end{array}$ & 0.05525 \\
\hline 7 & Idul Fitri I & $\begin{array}{c}- \\
1.1076963 \\
5\end{array}$ & $\begin{array}{c}- \\
1.2426000 \\
6\end{array}$ & $\begin{array}{c}0.1349037 \\
1\end{array}$ & $3 . \overline{-}$ & 2.258854 & $\begin{array}{c}- \\
3.77947 \\
9\end{array}$ & 2.671783 \\
\hline 8 & Idul Fitri II & $\begin{array}{c}- \\
1.4037836 \\
2 \\
\end{array}$ & $\begin{array}{c}0.8860522 \\
06\end{array}$ & $\begin{array}{c}2.2898358 \\
2 \\
\end{array}$ & $\begin{array}{c}- \\
2.09673 \\
7\end{array}$ & 0.692953 & $\begin{array}{c}- \\
1.13379 \\
5\end{array}$ & 0.26999 \\
\hline 9 & Wafatnya Yesus Kristus & $\begin{array}{l}0.6828415 \\
93\end{array}$ & $\begin{array}{c}2.0046369 \\
04\end{array}$ & $\begin{array}{c}1.3217953 \\
1\end{array}$ & 0.96 & 0.27716 & $\begin{array}{c}1.01909 \\
4\end{array}$ & 0.33625 \\
\hline 10 & Kenaikan Yesus Kristus & -0.7062478 & $\begin{array}{c}- \\
1.1598159 \\
4 \\
\end{array}$ & $\begin{array}{c}0.4535681 \\
41 \\
\end{array}$ & $\begin{array}{c}- \\
1.70364 \\
2\end{array}$ & 0.997394 & $\begin{array}{c}- \\
0.89444 \\
1\end{array}$ & 0.188194 \\
\hline 11 & Natal & $\begin{array}{l}2.9014656 \\
84\end{array}$ & $\begin{array}{c}2.0041220 \\
6\end{array}$ & $\begin{array}{c}0.8973436 \\
23\end{array}$ & $\begin{array}{c}1.68571 \\
53\end{array}$ & 1.21575 & $\begin{array}{c}2.05528 \\
08\end{array}$ & 0.846185 \\
\hline 12 & Nyepi & $\begin{array}{c}2.9175867 \\
94 \\
\end{array}$ & $\begin{array}{c}- \\
3.9973639 \\
4 \\
\end{array}$ & $\begin{array}{c}6.9149507 \\
32 \\
\end{array}$ & $\begin{array}{c}1.79171 \\
65\end{array}$ & 1.12587 & $\begin{array}{c}2.05219 \\
6 \\
\end{array}$ & 0.865391 \\
\hline 13 & Tahun Baru Imlek & $\begin{array}{c}- \\
3.0973818 \\
5 \\
\end{array}$ & $\begin{array}{c}- \\
4.9994811 \\
8 \\
\end{array}$ & $\begin{array}{c}1.9020993 \\
29\end{array}$ & -4.08 & 0.982618 & $\begin{array}{c}- \\
3.33133 \\
8\end{array}$ & 0.233956 \\
\hline 14 & Waisak & $\begin{array}{c}- \\
5.9735988 \\
6 \\
\end{array}$ & $\begin{array}{c}- \\
1.1609675 \\
7 \\
\end{array}$ & 4.8126313 & $\begin{array}{c}- \\
3.38509 \\
4\end{array}$ & 2.58851 & $\begin{array}{c}- \\
3.78215 \\
5\end{array}$ & 2.19144 \\
\hline & Average Percentage E & or (MAPE) & & $\begin{array}{c}1.8588413 \\
79\end{array}$ & & $\begin{array}{c}1.1557759 \\
29\end{array}$ & & $\begin{array}{c}\mathbf{0 . 9 7 5 5 2 3 8} \\
57\end{array}$ \\
\hline
\end{tabular}

TABEL VI. Results of Peak Load forecastingon National Holidaysin 2013

\begin{tabular}{|c|c|c|c|c|c|c|c|c|}
\hline \multirow[b]{2}{*}{ No } & \multirow[b]{2}{*}{ Holidays Name } & \multirow{2}{*}{$\begin{array}{l}\text { Actual } \\
(M W)\end{array}$} & \multicolumn{2}{|c|}{ IT2 } & \multicolumn{2}{|c|}{ IT2-BBBC } & \multicolumn{2}{|c|}{ IT2-FLOWER } \\
\hline & & & $\begin{array}{c}\text { Forecast } \\
(M W)\end{array}$ & Error(\%) & $\begin{array}{c}\text { Forecast } \\
(M W)\end{array}$ & $\begin{array}{l}\text { Absolute } \\
\text { Error(\%) }\end{array}$ & $\begin{array}{c}\text { Forecast } \\
(M W)\end{array}$ & Error(\%) \\
\hline 1 & Tahun Baru Masehi & $\begin{array}{c}15780.0 \\
0\end{array}$ & $\begin{array}{l}15870.8 \\
23\end{array}$ & 0.57556 & $\begin{array}{c}15870.8 \\
24\end{array}$ & 0.57556 & $\begin{array}{l}15914.4 \\
95\end{array}$ & 0.85232 \\
\hline 2 & $\begin{array}{l}\text { Proklamasi Kemerdekaan } \\
\text { RI }\end{array}$ & $\begin{array}{c}17354.0 \\
0 \\
\end{array}$ & $\begin{array}{c}17093.6 \\
6 \\
\end{array}$ & 1.5002 & $\begin{array}{c}16743.9 \\
43 \\
\end{array}$ & 3.5154 & $\begin{array}{c}17071.8 \\
14 \\
\end{array}$ & 1.6261 \\
\hline 3 & Idul Adha & $\begin{array}{c}18650.0 \\
0\end{array}$ & $\begin{array}{c}19071.9 \\
36\end{array}$ & 2.26239 & $\begin{array}{c}18621.7 \\
16\end{array}$ & 0.1517 & $\begin{array}{c}19103.5 \\
03\end{array}$ & 2.43165 \\
\hline 4 & Tahun Baru Hijriyah & $\begin{array}{c}19477.0 \\
0\end{array}$ & $\begin{array}{c}19583.1 \\
2\end{array}$ & 0.54485 & $\begin{array}{c}19486.7 \\
17\end{array}$ & 0.04989 & $\begin{array}{c}19463.5 \\
89\end{array}$ & 0.0689 \\
\hline 5 & $\begin{array}{l}\text { Maulid Nabi Muhammad } \\
\text { SAW }\end{array}$ & $\begin{array}{c}18307.0 \\
0\end{array}$ & $\begin{array}{l}17989.6 \\
9\end{array}$ & 1.7333 & $\begin{array}{c}17971.1 \\
15\end{array}$ & 1.8347 & $\begin{array}{c}18017.6 \\
97\end{array}$ & 1.5803 \\
\hline 6 & Isra Mi'raj & $\begin{array}{c}19071.0 \\
0\end{array}$ & $\begin{array}{c}18816.8 \\
12\end{array}$ & 1.3329 & 18999.9 & 0.3728 & $\begin{array}{c}19082.5 \\
29\end{array}$ & 0.06045 \\
\hline 7 & Idul Fitri I & $\begin{array}{c}13777.0 \\
0\end{array}$ & $\begin{array}{c}13754.8 \\
65\end{array}$ & 0.1607 & $\begin{array}{c}13406.3 \\
73\end{array}$ & 2.6902 & $\begin{array}{c}13338.6 \\
21\end{array}$ & 3.182 \\
\hline
\end{tabular}




\begin{tabular}{|c|c|c|c|c|c|c|c|c|}
\hline 8 & Idul Fitri II & $\begin{array}{c}14058.0 \\
0\end{array}$ & $\begin{array}{c}14413.3 \\
31\end{array}$ & 2.52761 & $\begin{array}{c}13950.4 \\
69\end{array}$ & 0.7649 & $\begin{array}{c}14099.8 \\
96\end{array}$ & 0.29802 \\
\hline 9 & Wafatnya Yesus Kristus & $\begin{array}{c}18853.0 \\
0\end{array}$ & $\begin{array}{c}19123.8 \\
23\end{array}$ & 1.4365 & $\begin{array}{c}18909.7 \\
87\end{array}$ & 0.30121 & $\begin{array}{c}18921.8 \\
95\end{array}$ & 0.36543 \\
\hline 10 & Kenaikan Yesus Kristus & $\begin{array}{c}19914.0 \\
0\end{array}$ & $\begin{array}{c}19818.3 \\
35\end{array}$ & 0.4804 & $\begin{array}{c}19703.6 \\
32\end{array}$ & 1.0564 & $\begin{array}{c}19874.3 \\
07\end{array}$ & 0.1993 \\
\hline 11 & Natal & $\begin{array}{c}18782.0 \\
0\end{array}$ & $\begin{array}{c}18602.7 \\
38\end{array}$ & 0.9544 & $\begin{array}{c}18539.1 \\
3\end{array}$ & 1.2931 & $\begin{array}{c}18612.9 \\
58\end{array}$ & 0.9 \\
\hline 12 & Nyepi & $\begin{array}{c}18723.0 \\
0\end{array}$ & $\begin{array}{c}17349.9 \\
85\end{array}$ & 7.3333 & $\begin{array}{c}18499.4 \\
5\end{array}$ & 1.194 & $\begin{array}{c}18551.1 \\
7\end{array}$ & 0.9177 \\
\hline 13 & Tahun Baru Imlek & $\begin{array}{c}17875.0 \\
0\end{array}$ & $\begin{array}{c}17488.3 \\
56\end{array}$ & 2.163 & $\begin{array}{c}17675.2 \\
61\end{array}$ & 1.1174 & $\begin{array}{c}17827.4 \\
43\end{array}$ & 0.2661 \\
\hline 14 & Waisak & $\begin{array}{c}18662.0 \\
0\end{array}$ & $\begin{array}{c}19700.2 \\
53\end{array}$ & 5.56346 & $\begin{array}{c}19220.4 \\
31\end{array}$ & 2.99234 & $\begin{array}{c}19134.7 \\
71\end{array}$ & 2.53334 \\
\hline \multicolumn{3}{|c|}{ Mean Average Percentage Error (MAPE) } & & $\begin{array}{c}2.0406121 \\
43\end{array}$ & & $\begin{array}{c}1.2792571 \\
43\end{array}$ & & $\begin{array}{c}1.0915435 \\
71\end{array}$ \\
\hline
\end{tabular}

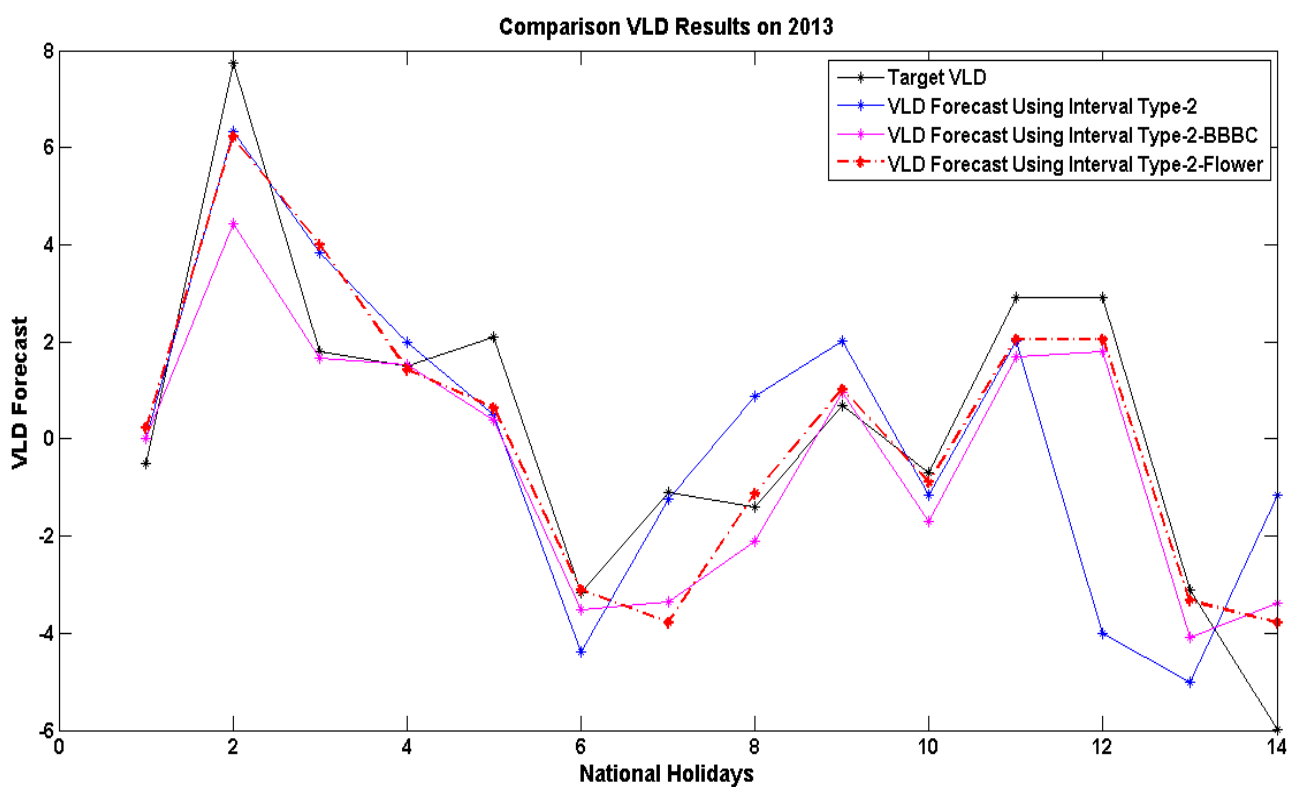

Fig9. Results of VLD Forecastingon National Holidays in 2013

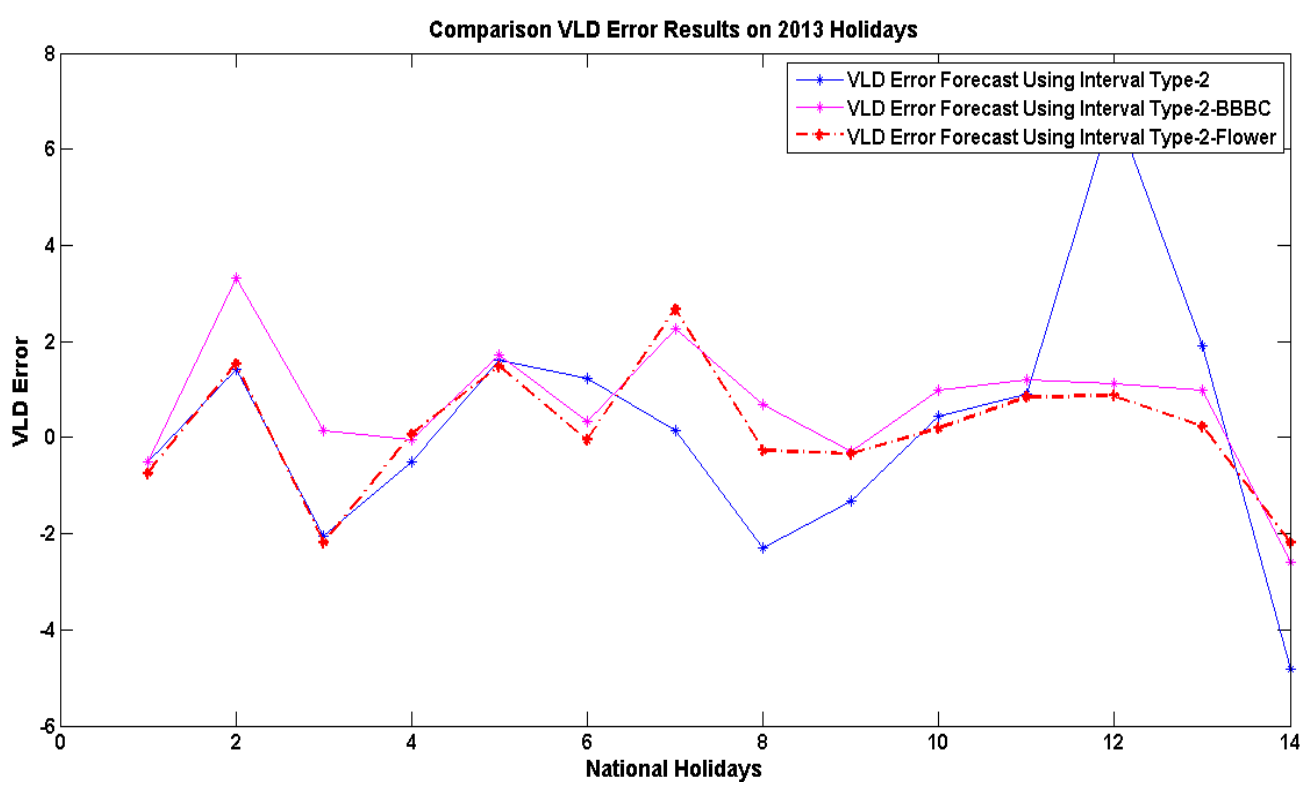

Fig10.Results of VLD Error Forecastingon National Holidays in 2013 


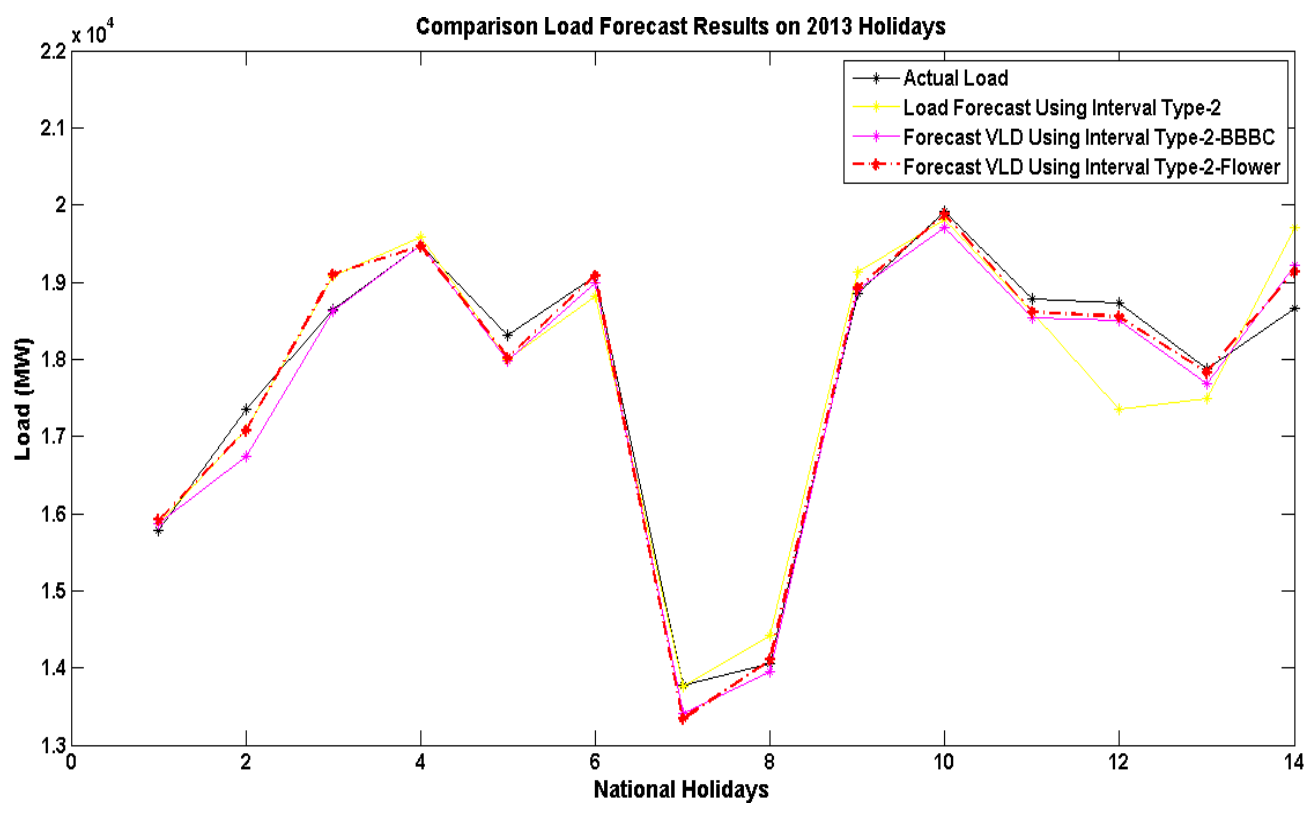

Fig11. Results of Load Forecast for National Holidays in 2013

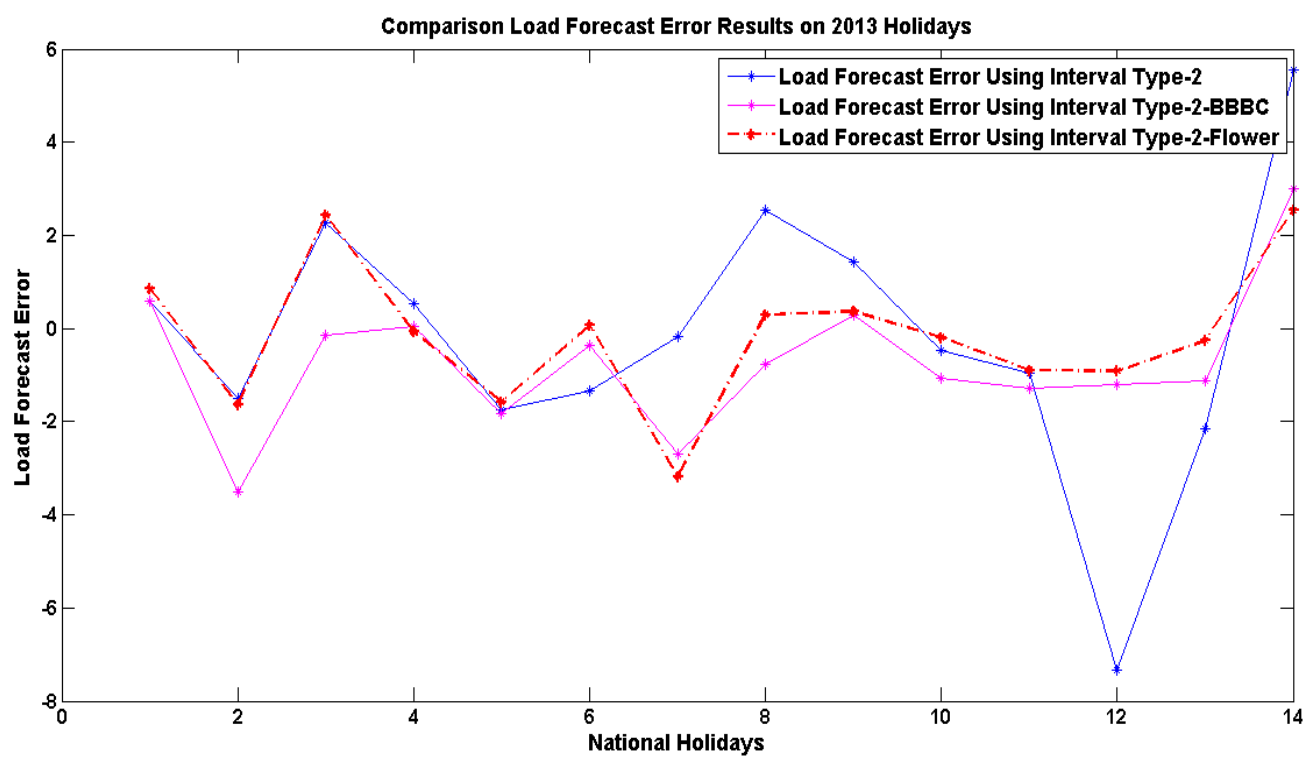

Fig12.Results of Load Forecasting Error onNational Holidays in 2013

\section{RESUlt AND ANALYSIS}

The calculation results of forecasting error Type-2 Fuzzy Logic-Flower Pollination Algorithm using data from various types of load conditions on holidays where this result is just a case of forecasting in 2008 show in Table 5 and 6. Figure 9-12 show the results of the plotting. Interval Type-2 Fuzzy Logic-Flower Pollination Algorithm (IT2FPA) method and several methods such as the Interval Type-2 Fuzzy Logic (IT2FL), Interval Type-2 Fuzzy Logic-Big Bang Big Crunch (IT2FL-BBBC) as a comparison.

The test results by using IT2FPA method as a proposed method for load forecasting have Mean Absolute Percentage Error (MAPE) is $1.091543571 \%$. By using IT2FL, MAPE is $2.040612143 \%$. By using IT2FLBBBC, MAPE is $1.279257143 \%$.

\section{Conclusions}

Interval Fuzzy Logic Type-2 method which is optimized by using Flower Pollination Algorithm proposed in this research can be used to forecast the peak load during some holidays in Jawa-Bali system, Indonesia.The method has MAPE which is less than $2 \%$. The method is very useful for operators to set up different scenarios for forecasting method.

\section{ACKNOWLEDGMENT}

Authors would like to thank Power System Operation and Control Laboratory, SepuluhNopember Institute of Technology (ITS Surabaya), for supporting this research. 


\section{REFERENCES}

[1] Mohammad Ghomi, Mahdi Goodarzi, and MahmoodGoodarzi,"Peak Load Forecasting of electric Utilities for West province of IRAN by usingNeural Network without Weather information”, IEEE International Conference on Computer Modelling and Simulation, 2010

[2] R. Weron, "Modeling and Forecasting Electricity Loads and Prices", England, John Wiley \& Sons Publisher, 2006.

[3] K-H Kim, H-SYoun, and Y-C Kang, "Short-Term Load Forecasting for Special Days in Anomalous Load Conditions Using Neural Networks and Fuzzy Inference Method”, IEEE Transactions On Power Systems, vol. 15, no. 2, pp. 559-565, May 2010.

[4] Young-Min Wi, Sung-Kwan Joo, and Kyung-Bin Song"Holiday Load Forecasting Using Fuzzy Polynomial Regression With WeatherFeature Selection and Adjustment," IEEE Trans. Power Syst., vol. 27, no. 2, pp. 596-603, May 2012.

[5] V. H. Hinojosaand A. Hoese, "Short-Term Load Forecasting Using Fuzzy Inductive Reasoning and Evolutionary Algorithms," IEEE Trans. Power Syst., vol. 25, no. 1, pp. 565-574, February 2010.

[6] A. Jain, and B. Satish, "Clustering based Short Term Load Forecasting using Artificial Neural Network",978-1-4244-3811-2/09 (C2009 IEEE.

[7] N.M. Pindoriya, S.N. Singh, and S.K. Singh, "One-Step-Ahead Hourly Load Forecasting Using Artificial Neural Network",IEEE International Conference on Power Systems, 2009.

[8] E. Banda and K. A. Folly, "Short Term Load Forecasting Using ArtificialNeural Network," IEEE PowerTech 2007. PP.108-112, 2007.

[9] M.M. Othman, M.H.H. Harun, and I. Musirin, "Short Term Load Forecasting Using Artificial Neural Network with FeatureExtraction Method and Stationary Output," IEEE Internationl Power Engineering and Optimization Conference, pp. 480 -484, June. 2012.

[10] Farah N, Khadir M.T., Bouaziz I. and Kennouche, "Short-term Forecasting of Algerian Load Using Fuzzy Logic And Expert System,’IEEE978-1-4244-3757-3/09@2009.

[11] Thiang and Y. Kurniawan, "Electrical Load Time Series Data Forecasting Using Interval Type-2 Fuzzy Logic System," IEEE 978-1$4244-5540-9 / 10 \odot 2010$, pp. $527-531,2010$

[12] A.Khosravi, S.Nahavandi, and D. Creighton, "Short Term Load Forecasting Using Interval Type-2 Fuzzy Logic Systems" IEEEInternational Conference on Fuzzy Systems, pp. 502 -508, June 2011

[13] A.Khosraviand S.Nahavandi, "Load Forecasting Using Interval Type-2 Fuzzy Logic Systems: Optimal Type Reduction," IEEE Transactions On Industrial Informatics, Vol. 10, No. 2, pp. 1055 -1063, May2014

[14] A. Imran, I M. Y. Negara, and I.Robandi, "Peak Load Forecasting On National Holiday Using Fuzzy-Firefly Algorithm at Jawa-Bali Electricity System In Indonesia,"International Review of Electrical Engineering (I.R.E.E.), (submitted)

[15] H. S.Hippert, C. E.Pedreira, and R. C. Souza, "Neural Networks for Short-Term Load Forecasting: A Review and Evaluation," IEEE Trans. Power Syst., vol. 16, no. 1, pp. $44-55$, February 2001

[16] P.Day, M. Fabian, D. Noble, G. Ruwisch, R. Spencer, J. Stevenson,and R.Thoppay, "Residential Power Load Forecasting," ELSEVIERConference on System Engineering Research, pp. 457 -464, March 2014

[17] A.Ramadhani, A. Dharmaand I. Robandi, "Optimization FOU of Interval Type-2 Fuz.zy Inference System Using Big Bang - Big Crunch Algorithm for Short Term Load Forecasting on National Holiday Case Study: South and Central KalimantanIndonesia",International Review of Electrical Engineering (I.R.E.E.), Vol. 10, N. 1, ISSN 1827- 6660, January-February 2015

[18] O. Castillo and P. Melin, "Recent Advances in Interval Type-2 Fuzzy Systems," SpringerBriefs in Computational Intelligence, DOI: 10.1007/978-3-642-28956-9 2, 2012

[19] X-S Yang, "Flower pollination algorithm for global optimization," Unconventional Computation and Natural Computation 2012, Lecture Notes inComputer Science, Vol. 7445, pp. 240-249 2012

\section{AUTHOR PROFILE}

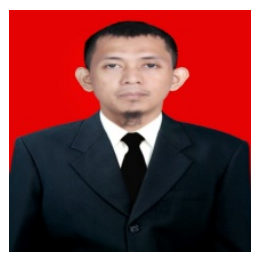

Andi Imran was born in Makassar in 1985. He received bachelor degree from State University of Makassar in Electrical Engineering, and then master degree from Hasanuddin University in Electrical Engineering. His research is about Power System Operation and Control especially in Load Forecasting using artificial intelligence method. Since 2013, he is astudent inElectrical Engineering Department at SepuluhNopemberInstitute of Technology (ITS Surabaya) in Indonesia. Email:andi.imran13@mhs.ee.its.ac.id

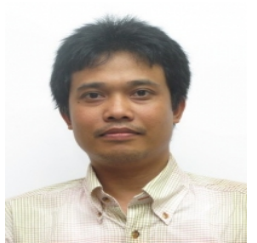

I Made Yulistya Negara was born in Bali, Indonesia on 12 July 1970. He received the B.Sc. and M.Sc. degrees in electrical engineering from Sepuluh Nopember Institute of Technology in Indonesia and Karlsruhe University in Germany in 1994 and 2001, respectively. From 1994 to 1997, he worked as a field engineer in Wijaya Karya Construction Co., Indonesia. From 2001 to 2003, he has been working as an Assistant Lecturer at Sepuluh Nopember Institute of Technology in Indonesia. Since 2004 he has been enrolled in doctoral program at Kyushu University, Fukuoka in Japan. He is a member of the Institute of Electrical Engineers, Japan (IEEJ). Email:yulistya@ee.its.ac.id.

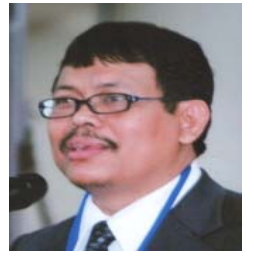

Imam Robandi received Doctor of Design and Information Engineering at Tottori University in Japan. Since 2008, he has been Professor in Electrical Engineering Department at SepuluhNopember Institute of Technology. His research is about Dynamic Stability problem of large scale electrical power system with intelligent control, optimization control, adaptive control and intelligent control. He has awarded for encounment of research and development from Institute of electrical engineer of Japan (IEEJ) in 2000. Email:robandi@ee.its.ac.id. 